\title{
Nontrivial temperature dependence of magnetic anisotropy in multiferroic $\mathrm{Ba}_{2} \mathrm{MnGe}_{2} \mathrm{O}_{7}$
}

\author{
Shunsuke Hasegawa $\odot,{ }^{1}$ Shohei Hayashida $\odot,{ }^{1}$ Shinichiro Asai, ${ }^{1}$ Masato Matsuura $\odot{ }^{2}$ \\ Zaliznyak Igor, ${ }^{3}$ and Takatsugu Masuda ${ }^{1,4,5}$ \\ ${ }^{1}$ Institute for Solid State Physics, The University of Tokyo, Chiba 277-8581, Japan \\ ${ }^{2}$ Neutron Science and Technology Center, Comprehensive Research Organization for Science and Society, Ibaraki 319-1106, Japan \\ ${ }^{3}$ Condensed Matter Physics and Materials Science Department, Brookhaven National Laboratory, Upton, New York 11973, USA \\ ${ }^{4}$ Institute of Materials Structure Science, High Energy Accelerator Research Organization, Ibaraki 305-0801, Japan \\ ${ }^{5}$ Trans-scale Quantum Science Institute, The University of Tokyo, Tokyo 113-0033, Japan
}

(Received 29 April 2021; accepted 8 July 2021; published 28 July 2021)

\begin{abstract}
We measured the temperature dependences of the static magnetization and the spin excitation in the squarelattice multiferroic $\mathrm{Ba}_{2} \mathrm{MnGe}_{2} \mathrm{O}_{7}$. An anisotropy gap of the observed low-energy mode is scaled by electric polarization rather than a power of the sublattice moment. Spin-nematic interaction in the effective spin Hamiltonian, which is equivalent to interaction of the electric polarization, is responsible for the easy-axis anisotropy. The nontrivial behavior of the anisotropy gap can be rationalized as change in the hybridized $d-p$ orbital with temperature, leading to the temperature dependence of the spin-nematic interaction.
\end{abstract}

DOI: 10.1103/PhysRevResearch.3.L032023

Spin-driven multiferroics [1-3] have been extensively studied since the discovery of an enhanced magnetoelectric (ME) effect in $\mathrm{TbMnO}_{3}$ [4]. Through spin-orbit coupling (SOC), a spin order induces a change in charge distribution, leading to the emergence of electric polarization. The microscopic mechanisms of the multiferroics are categorized into three types [1]: the spin current [5], the exchange striction [6], and the spin-dependent $d$-p hybridization [7,8]. A notable feature of the latter is that hybridized $d$ and $p$ orbitals of magnetic ion and ligand are modulated by spin states via SOC, and this induces an electric polarization. The relation between the electric polarization $\boldsymbol{P}$ and the spin moment $\boldsymbol{S}$ is locally described as $\boldsymbol{P}=\Lambda \sum_{i}\left(\boldsymbol{S} \cdot \boldsymbol{e}_{i}\right)^{2} \boldsymbol{e}_{i}$, where $\boldsymbol{e}_{i}$ is the bonding vector between the magnetic ion and the $i$ th ligand. Coefficient $\Lambda$ is determined by SOC and transfer integrals between the magnetic ion and ligands. The mechanism has been identified in $\mathrm{CuFeO}_{2}$ [7], åkermanite compounds [9-11], $\mathrm{Cu}_{2} \mathrm{OSeO}_{3}[12,13]$ and rare-earth ferroborates [14,15]. Since the direction of the local spin moment determines the direction of the electric polarization, magnetic anisotropy plays a key role in forming multiferroic structure, i.e., the simultaneous structures of the spin and polarization $[10,11,16]$. The magnitude of the anisotropy gives the energy scale for the control of the magnetism by the electric field as well as the magnetic field, which was tested in $\mathrm{Cu}_{2} \mathrm{OSeO}_{3}$ [13] and an åkermanite $\mathrm{Ba}_{2} \mathrm{CoGe}_{2} \mathrm{O}_{7}$ [17]. Although the temperature $T$ dependence of the anisotropy gap in the magnon spectrum in a conventional magnet scales as a power of the sublattice

Published by the American Physical Society under the terms of the Creative Commons Attribution 4.0 International license. Further distribution of this work must maintain attribution to the author(s) and the published article's title, journal citation, and DOI. magnetic moment $[18,19]$, this may not be the case for multiferroics. The change in the polarization with $T$ affects the spin-interaction parameters through $d$-p hybridization, which might lead to a nontrivial behavior in the $T$ dependence of the anisotropy and a low-energy spin dynamics near the magnetic $\Gamma$ point.

We focus on the multiferroic $\mathrm{Ba}_{2} \mathrm{MnGe}_{2} \mathrm{O}_{7}$ [20], which is isostructural to $\mathrm{Ba}_{2} \mathrm{CoGe}_{2} \mathrm{O}_{7}$ but is distinct in that the effect of the crystal electric field on the isotropic charge distribution of the half-filled shell of $\mathrm{Mn}^{2+}$ ion is small. Hence weak spin-nematic interaction originating from the $d$ - $p$ hybridization with the ligand, which can be sensitive to a small change in the orbitals with the temperature, is the main source of magnetic anisotropy. The crystal structure is tetragonal, and the space group is $P \overline{4} 2_{1} m$ [21], as shown in Fig. 1(a). $\mathrm{Mn}^{2+}$ ions carrying spin $S=5 / 2$ form a square lattice in the $a b$ plane. The magnetic susceptibility exhibits a typical behavior of a classical square-lattice Heisenberg antiferromagnet with an interaction of $26 \mu \mathrm{eV}$. At the Néel temperature $T_{\mathrm{N}}$ of $4 \mathrm{~K}$, a magnetic long-range order with a propagation vector of $(1,0,1 / 2)$ sets in, and a collinear spin structure in which the spins lie in the $a b$ plane is realized as shown in Fig. 1(b). The magnitude of the magnetic moment at $1.7 \mathrm{~K}$ is $4.66 \mu_{B}$ and is close to the saturation moment. The electric polarization appears below $T_{\mathrm{N}}$ when the magnetic field is applied along the crystallographic [110] direction [9], and the origin of the multiferroicity is explained by the spin-dependent $d$-p hybridization mechanism, which is also the main source of $\mathrm{Mn}^{2+}$ spin anisotropy. An inelastic neutron scattering (INS) experiment at $1.7 \mathrm{~K}$ using a conventional triple-axis spectrometer with an energy resolution of $0.1 \mathrm{meV}$ determined the main parameters in the presumed isotropic spin Hamiltonian; the nearest-neighbor interaction in the $a b$ plane $J_{1}$ is 27.8 $\mu \mathrm{eV}$, and the interplane interaction $J_{2}$ is $1.0 \mu \mathrm{eV}$. To reveal the magnetic anisotropy in the low-energy dynamics and its 


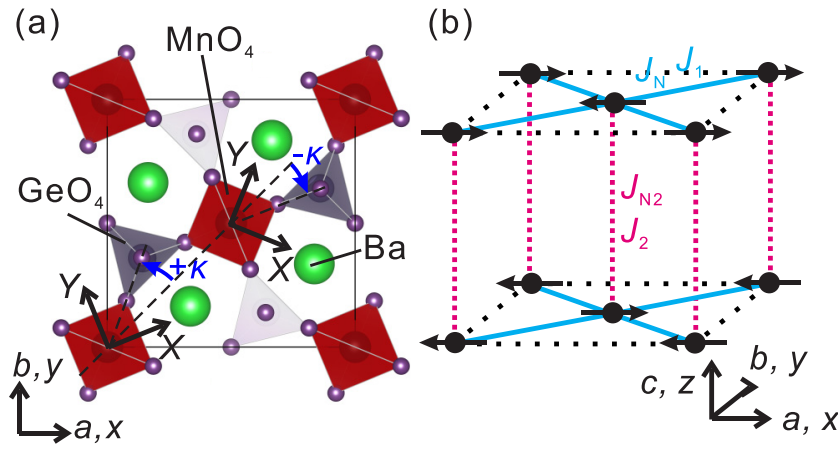

FIG. 1. (a) Crystal structure of $\mathrm{Ba}_{2} \mathrm{MnGe}_{2} \mathrm{O}_{7} \cdot \mathrm{MnO}_{4}$ tetrahedra are alternatively tilted by the characteristic angle of $\kappa$. (b) Magnetic structure of $\mathrm{Ba}_{2} \mathrm{MnGe}_{2} \mathrm{O}_{7}$ and the exchange pathways.

possible nontrivial behavior in the $T$ dependence, we study the detailed field-temperature $(H-T)$ phase diagram and inelastic neutron scattering spectra using a state-of-art spectrometer with ultrahigh resolution.

Single crystals were grown in an $\mathrm{O}_{2}$ atmosphere by the floating zone method. The magnetization was measured by a commercial superconducting quantum interference device (SQUID) magnetometer. The INS experiment was carried out using the near-backscattering spectrometer DNA in the Japan Proton Accelerator Research Complex (J-PARC) Materials and Life Science Experimental Facility (MLF) [22]. The horizontal scattering plane was the $a c$ plane, and the final energy of neutrons $E_{\mathrm{f}}$ was set to $2.084 \mathrm{meV}$ by using a $\mathrm{Si}(111)$ analyzer. The energy resolution was estimated to be $5.5 \mu \mathrm{eV}$ in FWHM at an elastic condition of incoherent scattering near $\boldsymbol{Q}=(1,0,1 / 2)$. A dilution refrigerator was used to cool the sample to $0.05 \mathrm{~K}$.

In the field derivative of the magnetization $d M / d H$, the fourfold rotational symmetry in the $a b$ plane which is a characteristic feature of the spin-nematic interaction as demonstrated in $\mathrm{Ba}_{2} \mathrm{CoGe}_{2} \mathrm{O}_{7}$ [11] is observed [Fig. 2(a)]. Enhancements of $d M / d H$ at $H=600$ Oe for $H \|$ [110] and [110] are due to a spin-flop (SF) transition. The existence of the biaxial SF transitions suggests that the easy axes are [100] and [010]. The corresponding electric polarization structure is antiferroelectric where $\boldsymbol{P}$ is along the $c$ axis [9]. The SF field drastically decreases with increasing $T$ as shown in the $H-T$ phase diagram in Fig. 2(c), which contrasts with conventional antiferromagnets exhibiting approximately $T$-independent behavior at $T<T_{\mathrm{N}}$.

Figure 3(a) shows the INS spectrum measured at $0.05 \mathrm{~K}$. Two dispersive modes with a boundary energy of $0.55 \mathrm{meV}$ and with gaps of $\sim 0.05 \mathrm{~m}$ and $\sim 0.1 \mathrm{meV}$ are clearly observed. The overall energy scale is consistent with a previous study [20]. It is remarkable that the two dispersive modes with finite gaps are clearly resolved, which was not the case in the previous study because of the relaxed energy resolution. Anisotropy gaps $\left(E_{g}\right)$ of the low- and high-energy modes, $\mathrm{T}_{1}$ and $\mathrm{T}_{2}$, respectively, at the magnetic $\Gamma$ point, $\boldsymbol{Q}=(1,0,1 / 2)$, are identified at 36 and $113 \mu \mathrm{eV}$. The latter is consistent with the value reported in a previous electron spin resonance (ESR) study [23]. $E_{g}$ of the $\mathrm{T}_{1}$ mode $\left(E_{g 1}\right)$ is drastically suppressed with the increase in $T$ as shown in Figs. 3(b)-3(e), while $E_{g}$ of

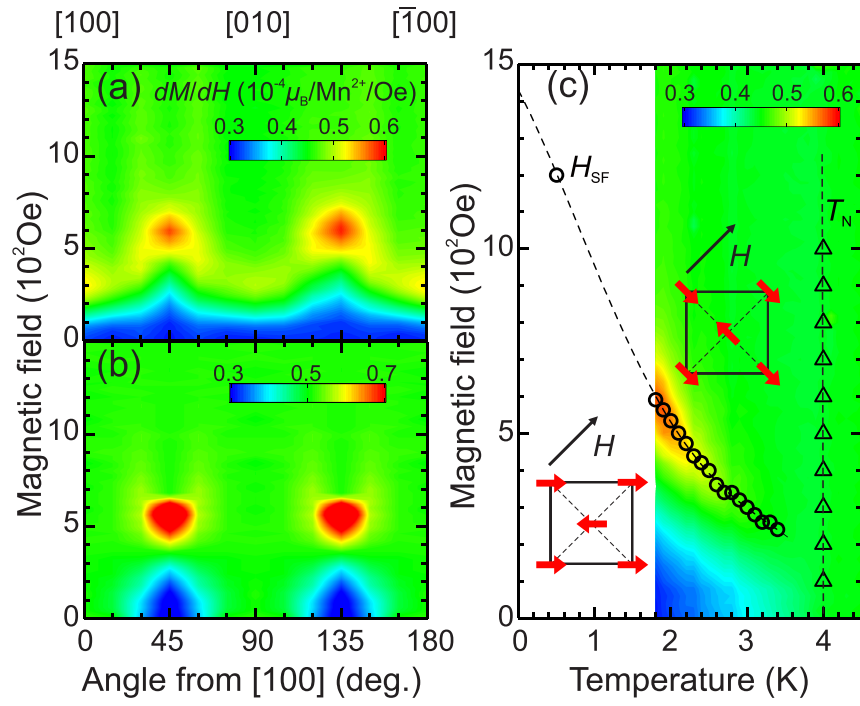

FIG. 2. Field derivative of magnetization $(d M / d H)$. (a) False color plot of $d M / d H$ measured in the field applied in the $a b$ plane at $1.8 \mathrm{~K}$. The horizontal axis is the angle between [100] and the field directions. (b) False color plot of calculated $d M / d H$ in the $a b$ plane. (c) Measured $H-T$ map of $d M / d H$ for $H \|$ [110]. $d M / d H$ curves measured at typical temperatures are shown in Supplemental Fig. S3. Black circles indicate $H_{\mathrm{SF}}$ determined by the peak tops of $d M / d H$. A circle at $0.5 \mathrm{~K}$ indicates $H_{\mathrm{SF}}$ reported in a previous study [20]. Triangles indicate Néel temperatures evaluated from the $T$ dependence of the magnetization. The representative descriptions of the magnetic structures below and above $H_{\mathrm{SF}}$ are inserted.

the $\mathrm{T}_{2}$ mode $\left(E_{g 2}\right)$ is moderately suppressed. The intensity of the modes at the $\Gamma$ point increases with increasing $T$ according to the thermal balance factor, as shown in Fig. 3(f).

The $T$ dependences of $E_{g 2}$ and the sublattice moment $g \mu_{\mathrm{B}}\langle S\rangle$ are shown in Fig. 4(a). The change in $E_{g 2}$ in the measurement range is small, and it scales as $g \mu_{\mathrm{B}}\langle S\rangle$. The behavior is consistent with conventional antiferromagnets where single-ion anisotropy is dominated by quadratic forms of spin operators $[18,19]$. In contrast, $E_{g 1}$ in Fig. 4(b) increases continuously with the decrease in $T$, and it cannot be scaled either by the sublattice moment or by any power of it. This indicates that the $T_{1}$ mode is not purely magnetic dynamics of electronic spin, but is hybridized with some other degrees of freedom.

We calculate the $d M / d H$ curves and the dynamical structure factor of neutron scattering using the leading-order $1 / S$ expansion of the following Hamiltonian:

$$
\begin{gathered}
\mathcal{H}=\sum_{i, j} J_{1} \boldsymbol{S}_{i} \cdot \boldsymbol{S}_{j}+\sum_{k, l} J_{2} \boldsymbol{S}_{k} \cdot \boldsymbol{S}_{l} \\
+\sum_{i}\left\{D\left(S_{i}^{z}\right)^{2}+g \mu_{B} \boldsymbol{S}_{i} \cdot \boldsymbol{H}\right\}+\mathcal{H}_{\mathrm{N}} \\
\mathcal{H}_{\mathrm{N}}=\sum_{i, j} J_{\mathrm{N}} O_{i}^{X Y} O_{j}^{X Y}
\end{gathered}
$$

where $\quad O_{i}^{X Y}=\cos \left(2 \kappa_{i}\right)\left(S_{i}^{x} S_{i}^{y}+S_{i}^{y} S_{i}^{x}\right)-\sin \left(2 \kappa_{i}\right)\left\{\left(S_{i}^{x}\right)^{2}-\right.$ $\left.\left(S_{i}^{y}\right)^{2}\right\}$. The $x, y$, and $z$ axes in the Hamiltonian (1) are along the crystallographic $a, b$, and $c$ axes, respectively, as shown in Fig. 1(b). $\kappa_{i}$ is a tilt angle of the $\mathrm{MnO}_{4}$ tetrahedron from 

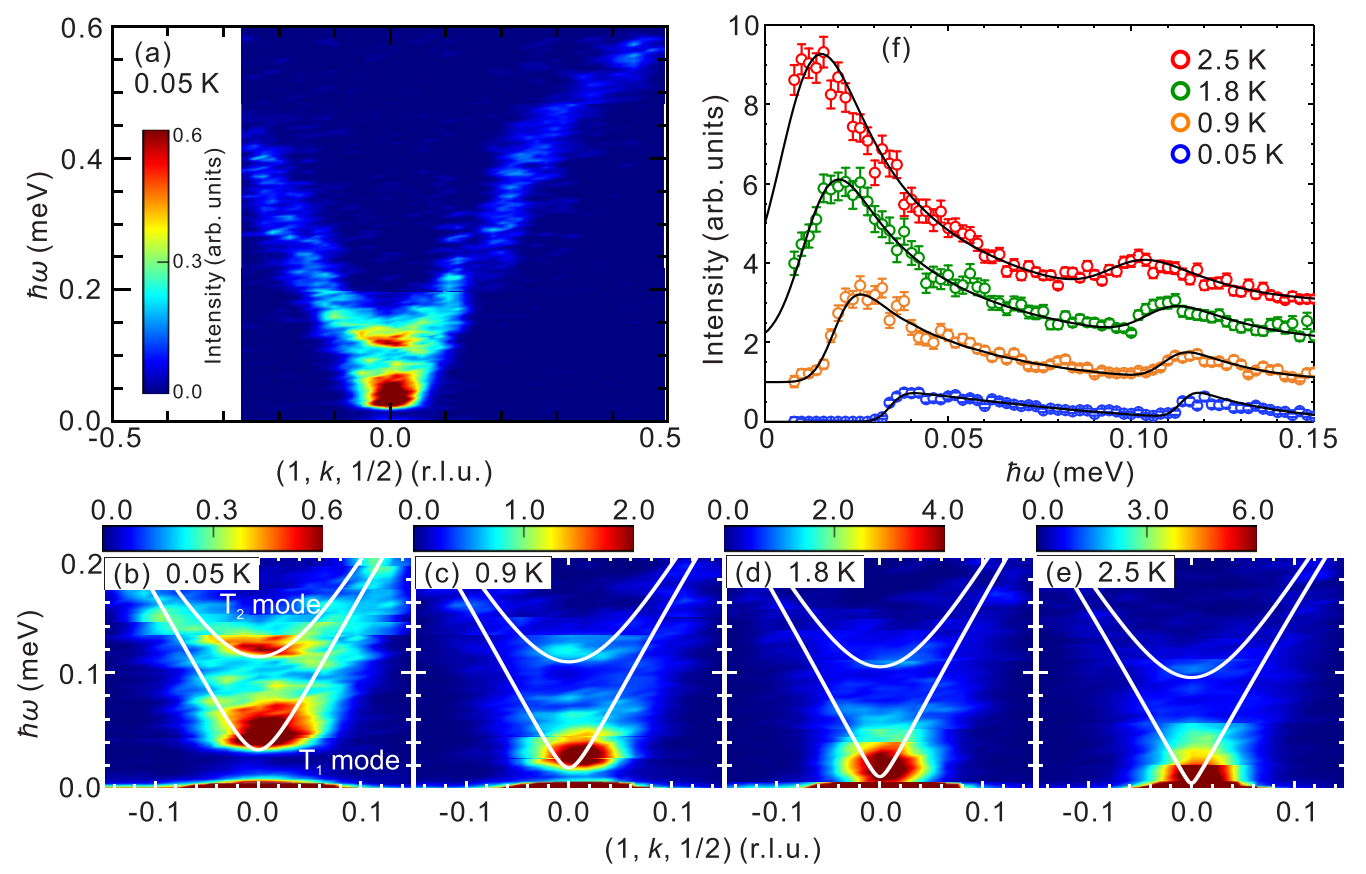

FIG. 3. Inelastic neutron scattering (INS) spectra. (a) False color plot of the INS spectrum measured at $0.05 \mathrm{~K}$ projected onto the $\hbar \omega-(1, k, 1 / 2)$ plane. The spectrum is integrated in the range of $0.9 \leqslant h \leqslant 1.1$ and $0.45 \leqslant l \leqslant 0.55$. (b)-(e) The INS spectra focused on the low-energy range measured at (b) $0.05 \mathrm{~K}$, (c) $0.9 \mathrm{~K}$, (d) $1.8 \mathrm{~K}$, and (e) $2.5 \mathrm{~K}$. White solid curves are calculated dispersions. (f) Temperature evolution of constant- $\boldsymbol{q}$ cuts at $\boldsymbol{Q}=(1,0,1 / 2)$; data at different temperature are vertically offset. Coherent elastic scattering is subtracted for each temperature. The spectra are integrated in the range of $-0.035 \leqslant k \leqslant 0.035$. The asymmetric peak shape results from the wave vector resolution. Black solid curves are the calculated magnon cross section.

the crystallographic [110] direction as shown in Fig. 1(a). The first and the second terms are the nearest-neighbor intraand interplane interactions of the square lattice, respectively. The third term is a single-ion anisotropy of an easy-plane type, with $D>0$, and the fourth is a Zeeman term. $\mathcal{H}_{\mathrm{N}}$ is the spin-nematic interaction, which produces the biaxial anisotropy in the $a b$ plane $[10,11]$. Note that a single-ion anisotropy with the second order of spin operators is not allowed in the $a b$ plane due to the fourfold rotational symmetry. The lowest order in-plane single-ion anisotropy is of the fourth order, and the reason it is not included is explained at the end of Sec. I of the Supplemental Material [24]. Figure 2(b) shows the calculated angle dependence of $d M / d H$ using $J_{\mathrm{N}}=0.12 \mathrm{neV}$ and $g \mu_{\mathrm{B}}\langle S\rangle=4.66 \mu_{\mathrm{B}}$, which reasonably reproduces the experimental data at $1.8 \mathrm{~K}$ in Fig. 2(a). By using $H_{\mathrm{SF}}$ and $g \mu_{\mathrm{B}}\langle S\rangle$ measured at each temperature we can estimate an effective $J_{\mathrm{N}}(T)$. We find that $J_{\mathrm{N}}$ as well as $H_{\mathrm{SF}}$ is strongly dependent on $T$ [Supplemental Fig. S5] and it cannot be explained solely by the change in $g \mu_{\mathrm{B}}\langle S\rangle$. The details of the calculation are described in Secs. I and II of the Supplemental Material [24].

Based on the spin-wave calculation, the gap energies of the magnetic anisotropy at the magnetic $\Gamma$ point are $\Delta_{1}^{\mathrm{SWT}} \sim 16\langle S(T)\rangle^{2} \sqrt{J_{\mathrm{N}}(T)\left(2 J_{1}+J_{2}+D / 2\right)}$ for the $\mathrm{T}_{1}$ mode and $\Delta_{2}^{\mathrm{SWT}}=4\langle S(T)\rangle \sqrt{D\left(2 J_{1}+J_{2}\right)}$ for the $\mathrm{T}_{2}$ mode. $\Delta_{1}^{\mathrm{SWT}}$ can be calculated by using the values of $J_{\mathrm{N}}(T)$ from the $d M / d H$ curve and $\langle S(T)\rangle$ from the neutron scattering experiment in the present study and those of $J_{1}, J_{2}$, and $g$ in a previous study [20]. The obtained $T$ dependence of $\Delta_{1}^{\mathrm{SWT}}$ in Fig. 4(b), however, is not consistent with $E_{g 1}$, particularly in the low- $T$ region. Here, we note that a $\mathrm{Mn}$ atom has a nuclear spin $I=5 / 2$ and that the hyperfine coupling between nuclear and electron spins induces a low-energy gap [25] in addition to the magnetic anisotropy gap. The energy gap induced by the hyperfine coupling has no $\boldsymbol{q}$ dependence. Then the magnon dispersion relation is modified; $\left[\hbar \omega_{i}(\boldsymbol{q})\right]^{2}=\left[\hbar \omega_{i}^{\mathrm{SWT}}(\boldsymbol{q})\right]^{2}+$ $\left(\Delta^{\mathrm{HF}}\right)^{2}$, where $\hbar \omega_{i}^{\mathrm{SWT}}(\boldsymbol{q})$ is the pure magnon dispersion for the $\mathrm{T}_{i}$ mode $(i=1,2)$ and $\Delta^{\mathrm{HF}}$ is the hyperfine gap [26]. The two gaps $E_{g 1}$ and $E_{g 2}$ at $Q=(1,0,1 / 2)$ are described as $E_{g 1}^{2}=\left(\Delta_{1}^{\mathrm{SWT}}\right)^{2}+\left(\Delta^{\mathrm{HF}}\right)^{2}$ and $E_{g 2}^{2}=\left(\Delta_{2}^{\mathrm{SWT}}\right)^{2}+\left(\Delta^{\mathrm{HF}}\right)^{2}$, where $\Delta^{\mathrm{HF}}=\sqrt{\left(\chi_{n}(T) / \chi_{c}\right)} A g \mu_{B}\langle S(T)\rangle$. Here, $\chi_{n}(T)$ is the paramagnetic nuclear spin susceptibility following the Curie law, $\chi_{c}$ is the electron spin susceptibility along the crystallographic $c$ direction, and $A$ is the hyperfine coupling. In order to estimate $\Delta^{\mathrm{HF}}$ and $D$, we fit the constant $\boldsymbol{q}$ cut in Fig. 3(f) to the calculated magnon cross section. The fit to the data is good as shown by black solid curves. The $T_{1}$ and $T_{2}$ modes in Figs. 3(b)-3(e) are reasonably reproduced by the calculated magnon dispersions shown by the white curves. The obtained hyperfine coupling constant is $A=240 \mathrm{kOe}$ as described in Sec. IV of the Supplemental Material [24], and it is consistent with Ref. [26] and the value reported in ${ }^{55} \mathrm{Mn}^{2+}$ ions in $\mathrm{Mn}$ diluted $\mathrm{ZnF}_{2}$ [27]. The $\mathrm{T}_{1}$ mode is, thus, hybridized with the nuclear spin. The $T$ dependence of $\Delta^{\mathrm{HF}}$ is shown in Figs. 4(a) and 4(b), and that of $\Delta_{2}^{\text {SWT }}$ is shown in Fig. 4(a).

$T$ dependences of $\Delta_{1}^{\mathrm{SWT}}$, a scaled electric polarization $P$ [9], and the second power of the scaled sublattice moment $\left(g \mu_{\mathrm{B}}\langle S\rangle\right)^{2}$ are shown in Fig. 4(c). We find that $\Delta_{1}^{\mathrm{SWT}}$ does not scale as $\left(g \mu_{\mathrm{B}}\langle S\rangle\right)^{2}$, but is rather consistent with the temperature dependence of electric polarization 


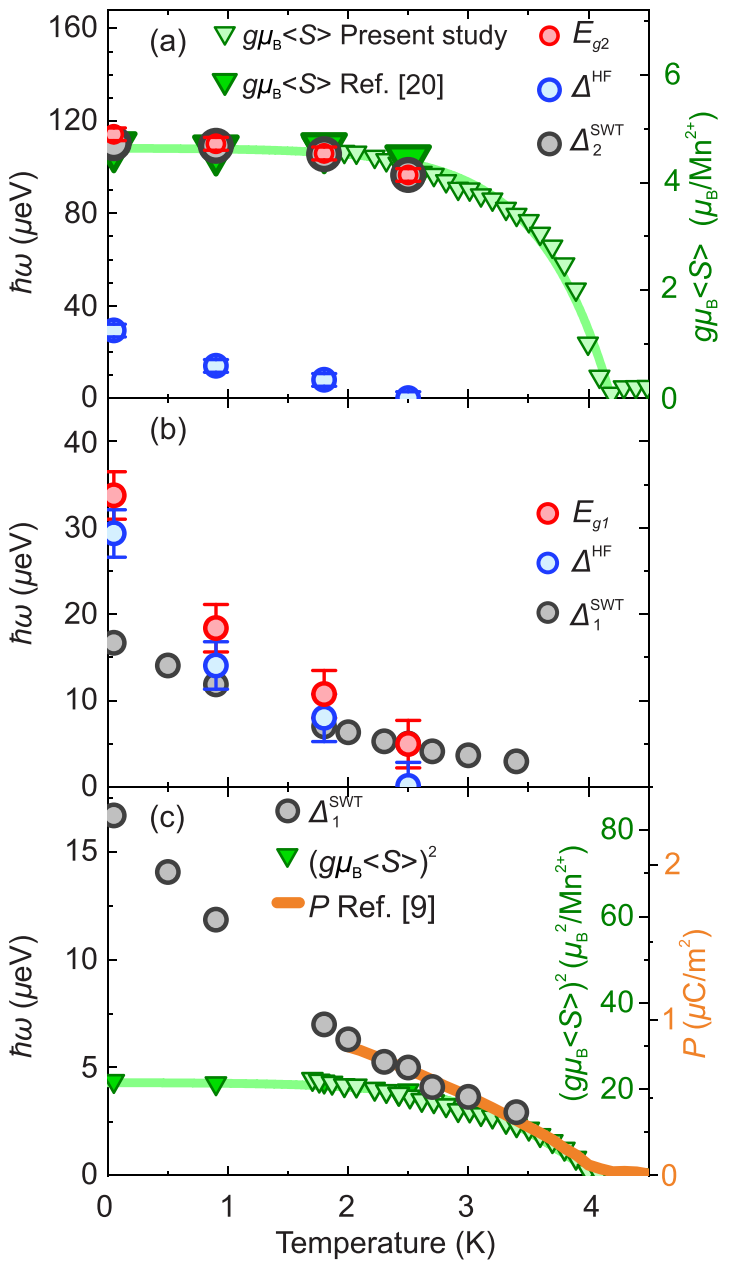

FIG. 4. The temperature dependences of gap energies observed in INS spectra, anisotropy energies, sublattice moment, and electric polarization. (a) $T$ dependences of $E_{g 2}$ (red circles), $\Delta_{2}^{\text {SWT }}$ (black circles), $\Delta^{\mathrm{HF}}$ (blue circles), and sublattice moment (light green triangles from a previous [20] study and dark green ones from the present study). $E_{g 2}, \Delta_{2}^{\mathrm{SWT}}$, and the sublattice moment are superimposed. The light green curve is a guide to the eye. (b) $T$ dependences of $E_{g 1}$ (red circles), $\Delta_{1}^{\mathrm{SWT}}$ (black circles), and $\Delta^{\mathrm{HF}}$ (blue circles). (c) $T$ dependences of $\Delta_{1}^{\mathrm{SWT}}$ (black circles), electric polarization $P$ (orange curve from Ref. [9]), and the second power of the sublattice moment (green triangles). The sublattice moment in this panel is a combination of measurements from the present and previous [20] studies in (a). Vertical error bars for $E_{g 1}, E_{g 2}$, and $\Delta^{\mathrm{HF}}$ in (a) and (b) indicate the experimental resolution of the spectrometer estimated at an elastic condition.

$P$. To discuss the behavior, we rewrite the formula of $\mathcal{H}_{\mathrm{N}}$ in Eq. (2) in terms of $P$. From the local relation between $\boldsymbol{S}$ and $\boldsymbol{P}$, one has $P_{i}^{Z}=\Lambda_{Z} O_{i}^{X Y}$ [10,11]. This relation leads to $\mathcal{H}_{\mathrm{N}}=\sum_{i, j} J_{\mathrm{N}} O_{i}^{X Y} O_{j}^{X Y}=\sum_{i, j} J_{P} P_{i}^{Z} P_{j}^{Z}$, where $J_{P}$ is an effective interaction between the electric polarizations; $J_{P}=J_{\mathrm{N}} / \Lambda_{Z}^{2}$. By considering the structures of the spin and electric polarization at $T<T_{\mathrm{N}}$, we obtain $\Delta_{1}^{\mathrm{SWT}}=$ $16\langle P(T)\rangle \sqrt{J_{P}\left(2 J_{1}+J_{2}+1 / 2 D\right)} / \sin (2 \kappa)$. The fact that the magnetic anisotropy gap $\Delta_{1}^{\mathrm{SWT}}(T)$ is scaled by the electric polarization $P(T)$ means that $P$ induces an emergent magnetic field. In addition, we notice that $J_{P}$ is $T$ independent. Drastic
$T$ dependence of $J_{\mathrm{N}}$ thus results from the change in $\Lambda_{Z}$ with $T$; the modification of the hybridized orbitals of $\mathrm{Mn}^{2+}$ and $\mathrm{O}^{2-}$ ions with $T$ leads to a small change in the energy, which is negligible in a highly anisotropic system but is enhanced in a nearly isotropic system such as the symmetric half-filled shell of a $\mathrm{Mn}^{2+}$ ion, and it is probed as the change in $J_{\mathrm{N}}$ through SOC. Since the change in the hybridized $d-p$ orbital with $T$ explicitly affects the anisotropy gap of the acoustic magnon, we call it a hybridized magnon with a $d-p$ orbital. This is a characteristic quasiparticle of the multiferroics originating from the hybridization of spin and orbital momenta.

In contrast to $\Delta_{1}^{\mathrm{SWT}}$, the $T$ dependence of $\Delta_{2}^{\mathrm{SWT}}$ is almost flat at $T \leqslant 2.5 \mathrm{~K}$, and the scaled $\Delta_{2}^{\mathrm{SWT}}$ is proportional to $g \mu_{\mathrm{B}}\langle S\rangle$ as shown in Fig. 4(a). This is because the single-ion anisotropy $D$ is robust to the change in the orbitals with $T$ and it is essentially temperature independent. This is consistent with the dependence $\Delta_{2}^{\mathrm{SWT}}=4\langle S(T)\rangle \sqrt{D\left(2 J_{1}+J_{2}\right)}$ describing conventional antiferromagnets in which a singleion anisotropy is represented by the quadratic form of the spin operator $[18,19]$.

Now we compare the electric dipole energy of $\mathrm{Ba}_{2} \mathrm{MnGe}_{2} \mathrm{O}_{7}$ as a dielectric with that as a multiferroic. The former energy is estimated by the formula of the classical electromagnetism, $\quad U_{(1,2)}=1 /\left(4 \pi \epsilon_{0} \epsilon r^{3}\right)\left[\boldsymbol{P}_{1} \cdot \boldsymbol{P}_{2}-3\left(\boldsymbol{P}_{1}\right.\right.$. $\left.\hat{\boldsymbol{r}})\left(\boldsymbol{P}_{2} \cdot \hat{\boldsymbol{r}}\right)\right]$. The reported electric polarization is $\left(0,0, p_{c}\right)$ with $p_{c} \sim 0.8 \mu \mathrm{C} / \mathrm{m}^{2}$ at $2.0 \mathrm{~K}$ in a magnetic field of $1 \mathrm{~T}$ applied along the [110] direction [9]. The dielectric permittivity is $\epsilon \sim 14$ [23]. We assume that the polarization is localized at each $\mathrm{Mn}^{2+}$ ion. By summing up the pairs of the nearest-neighbor $\mathrm{Mn}^{2+}$ ions in the $a b$ plane and those along the $c$ axis, $U_{(1,2)}$ per $\mathrm{Mn}^{2+}$ ion is estimated to be $-0.54 \mathrm{peV}$. Next we estimate the electric dipole energy as a multiferroic system, where both dielectric and magnetic energies are renormalized. In $\mathrm{Ba}_{2} \mathrm{MnGe}_{2} \mathrm{O}_{7}$ the spin-nematic operator is equivalent to the electric polarization, and only the component $P_{i}^{Z}=\Lambda_{Z} O_{i}^{X Y}$ is active in the ordered state. Then the renormalized dipole energy is $E_{(1,2)}=J_{P} P_{1}^{Z} P_{2}^{Z}=J_{N} O_{1}^{X Y} O_{2}^{X Y}$ at $1.8 \mathrm{~K}$ by using $J_{\mathrm{N}}$ and $g \mu_{\mathrm{B}}\langle S\rangle$ obtained in the present study. Note that the spin moment points along the $a$ or $b$ direction in the ordered state and $E_{(1,2)}$ per $\mathrm{Mn}^{2+}$ ion is estimated to be $-7.95 \mathrm{neV}$. The renormalized dipole energy in $\mathrm{Ba}_{2} \mathrm{MnGe}_{2} \mathrm{O}_{7}$ is gigantic compared with the classical dipole energy. For the control of the electric polarization, the required electric field is much stronger than that estimated from classical electromagnetism. The electric polarization in spin-driven multiferroics is, thus, robust to the external electric field.

In conclusion, we found that the temperature dependence of the biaxial magnetic anisotropy energy in the multiferroic $\mathrm{Ba}_{2} \mathrm{MnGe}_{2} \mathrm{O}_{7}$ is scaled by the electric polarization. The change in the hybridized orbitals of the metal and the ligands with the temperature determines the spin-nematic interaction, leading to nontrivial temperature dependence of the anisotropy energy in the neutron spectrum as well as that of the spin-flop field. In contrast, conventional single-ion anisotropy energy of easy-plane type is independent of temperature. The effect of hyperfine coupling between nuclear and electron spins is also observed in the low-temperature region. A thorough accounting for this effect is important for accurate understanding of the temperature evolution of the electronic spin gap. 
We are grateful to T. Asami for supporting us in the neutron scattering experiment. The neutron experiment at the Materials and Life Science Experimental Facility of JPARC was performed under a user program (Proposal No. 2016B0140). S. Hasegawa and S. Hayashida were supported by the Japan Society for the Promotion of Science through the Leading Graduate Schools (MERIT). This project is supported by JSPS KAKENHI Grants No. JP19KK0069 and No. JP20K20896 and by the U.S.-Japan collaboration program.
[1] Y. Tokura, S. Seki, and N. Nagaosa, Multiferroics of spin origin, Rep. Prog. Phys. 77, 076501 (2014).

[2] S. Dong, J.-M. Liu, S.-W. Cheong, and Z. Ren, Multiferroic materials and magnetoelectric physics: symmetry, entanglement, excitation, and topology, Adv. Phys. 64, 519 (2015).

[3] M. Fiebig, T. Lottermoser, D. Meier, and M. Trassin, The evolution of multiferroics, Nat. Rev. Mater. 1, 16046 (2016).

[4] T. Kimura, T. N. Goto, H. Shintani, K. Ishizaka, T. Arima, and Y. Tokura, Magnetic control of ferroelectric polarization, Nature (London) 426, 55 (2003).

[5] H. Katsura, N. Nagaosa, and A. V. Balatsky, Spin Current and Magnetoelectric Effect in Noncollinear Magnets, Phys. Rev. Lett. 95, 057205 (2005).

[6] S.-W. Cheong and M. Mostovoy, Multiferroics: a magnetic twist for ferroelectricity, Nat. Mater. 6, 13 (2007).

[7] T.-h. Arima, Ferroelectricity induced by proper-screw type magnetic order, J. Phys. Soc. Jpn. 76, 073702 (2007).

[8] C. Jia, S. Onoda, N. Nagaosa, and J. H. Han, Bond electronic polarization induced by spin, Phys. Rev. B 74, 224444 (2006).

[9] H. Murakawa, Y. Onose, S. Miyahara, N. Furukawa, and Y. Tokura, Comprehensive study of the ferroelectricity induced by the spin-dependent $d$ - $p$ hybridization mechanism in $\mathrm{Ba}_{2} X \mathrm{Ge}_{2} \mathrm{O}_{7}(X=\mathrm{Mn}, \mathrm{Co}$, and $\mathrm{Cu})$, Phys. Rev. B 85, 174106 (2012).

[10] J. Romhányi, M. Lajkó, and K. Penc, Zero- and finitetemperature mean field study of magnetic field induced electric polarization in $\mathrm{Ba}_{2} \mathrm{CoGe}_{2} \mathrm{O}_{7}$ : Effect of the antiferroelectric coupling, Phys. Rev. B 84, 224419 (2011).

[11] M. Soda, M. Matsumoto, M. Månsson, S. Ohira-Kawamura, K. Nakajima, R. Shiina, and T. Masuda, Spin-Nematic Interaction in the Multiferroic Compound $\mathrm{Ba}_{2} \mathrm{CoGe}_{2} \mathrm{O}_{7}$, Phys. Rev. Lett. 112, 127205 (2014).

[12] S. Seki, X. Z. Yu, S. Ishiwata, and Y. Tokura, Observation of skyrmions in a multiferroic material, Science 336, 198 (2012).

[13] J. S. White, K. Prša, P. Huang, A. A. Omrani, I. Živković, M. Bartkowiak, H. Berger, A. Magrez, J. L. Gavilano, G. Nagy, J. Zang, and H. M. Rønnow, Electric-Field-Induced Skyrmion Distortion and Giant Lattice Rotation in the Magnetoelectric Insulator $\mathrm{Cu}_{2} \mathrm{OSeO}_{3}$, Phys. Rev. Lett. 113, 107203 (2014).

[14] A. I. Popov, D. I. Plokhov, and A. K. Zvezdin, Quantum theory of magnetoelectricity in rare-earth multiferroics: $\mathrm{Nd}, \mathrm{Sm}$, and Eu ferroborates, Phys. Rev. B 87, 024413 (2013).

[15] T. Kurumaji, K. Ohgushi, and Y. Tokura, Magnetoelectric responses from the respective magnetic $R$ and $\mathrm{Fe}$ subsystems in the noncentrosymmetric antiferromagnets $R \mathrm{Fe}_{3}\left(\mathrm{BO}_{3}\right)_{4}(R=$ Eu, Gd, and Tb), Phys. Rev. B 89, 195126 (2014).

[16] S. Hayashida, M. Soda, S. Itoh, T. Yokoo, K. Ohgushi, D. Kawana, H. M. Rønnow, and T. Masuda, Magnetic model in multiferroic $\mathrm{NdFe}_{3}\left(\mathrm{BO}_{3}\right)_{4}$ investigated by inelastic neutron scattering, Phys. Rev. B 92, 054402 (2015).

[17] M. Soda, S. Hayashida, B. Roessli, M. Månsson, J. S. White, M. Matsumoto, R. Shiina, and T. Masuda, Continuous control of local magnetic moment by applied electric field in multiferroics $\mathrm{Ba}_{2} \mathrm{CoGe}_{2} \mathrm{O}_{7}$, Phys. Rev. B 94, 094418 (2016).

[18] F. M. Johnson and A. H. Nethercot, Antiferromagnetic resonance in $\mathrm{MnF}_{2}$, Phys. Rev. 114, 705 (1959).

[19] R. J. Birgeneau, J. Skalyo, and G. Shirane, Critical magnetic scattering in $\mathrm{K}_{2} \mathrm{NiF}_{4}$, Phys. Rev. B 3, 1736 (1971).

[20] T. Masuda, S. Kitaoka, S. Takamizawa, N. Metoki, K. Kaneko, K. C. Rule, K. Kiefer, H. Manaka, and H. Nojiri, Instability of magnons in two-dimensional antiferromagnets at high magnetic fields, Phys. Rev. B 81, 100402(R) (2010).

[21] A. Sazonov, V. Hutanu, M. Meven, G. Roth, R. Georgii, T. Masuda, and B. Náfrádi, Crystal structure of magnetoelectric $\mathrm{Ba}_{2} \mathrm{MnGe}_{2} \mathrm{O}_{7}$ at room and low temperatures by neutron diffraction, Inorg. Chem. 57, 5089 (2018).

[22] K. Shibata, N. Takahashi, Y. Kawakita, M. Matsuura, T. Yamada, T. Tominaga, W. Kambara, M. Kobayashi, Y. Inamura, T. Nakatani, K. Nakajima, and M. Arai, The performance of TOF near backscattering spectrometer DNA in MLF, J-PARC, JPS Conf. Proc. 8, 036022 (2015).

[23] Y. Iguchi, Y. Nii, M. Kawano, H. Murakawa, N. Hanasaki, and Y. Onose, Microwave nonreciprocity of magnon excitations in the noncentrosymmetric antiferromagnet $\mathrm{Ba}_{2} \mathrm{MnGe}_{2} \mathrm{O}_{7}$, Phys. Rev. B 98, 064416 (2018).

[24] See Supplemental Material at http://link.aps.org/supplemental/ 10.1103/PhysRevResearch.3.L032023 for details of the spinwave calculation, field derivative of magnetization, analysis of inelastic neutron scattering spectra, and temperature dependence of $\Delta^{\mathrm{HF}}$

[25] A. S. Borovik-Romanov and V. Tulin, Mixed electron-nuclear resonance in antiferromagnetic $\mathrm{MnCO}_{3}$, JETP Lett. 1, 134 (1965).

[26] I. A. Zaliznyak, N. N. Zolin, and S. V. Petrov, Investigation of the hyperfine interaction in the antiferromagnetic $\mathrm{CsMnI}_{3}$, JETP Lett. 64, 473 (1996).

[27] A. M. Clogston, J. P. Gordon, V. Jaccarino, M. Peter, and L. R. Walker, Hfs of $\mathrm{F}^{19}$ in the electron paramagnetic resonance of Mn:ZnF 2 , Phys. Rev. 117, 1222 (1960). 